\section{Association of Candida species with Oral Submucous Fibrosis and Oral Leukoplakia: A Case Control study}

\section{Chandramani More', Ruchita Peter ${ }^{1}$, Gavli Nishma ${ }^{1}$, Youbai Chen ${ }^{2}$ and Naman Rao ${ }^{3 *}$}

1 Department of Oral Medicine and Maxillofacial Radiology, K.M. Shah Dental College and Hospital, Sumandeep Vidyapeeth University, Gujarat, India

\section{Abstract}

Introduction: Oral Submucous fibrosis (OSMF) and Oral Leukoplakia (OL) are the most common Potentially Malignant Disorders (OPMD's) seen in South Asia and South East Asian countries. A significant percentage of oral squamous carcinomas develop from pre-existing OPMD's- Candida organisms is thought to be associated with these lesions.

Aim: To determine the incidence and association of candida species in OSMF and OL.

Methods: A Case control study was undertaken in 150 participants, which were equally divided into three groups. The smear or swab taken were subjected for Gram staining and Sabouraud's dextrose agar. The Positive cultured Candida samples were analyzed by using HiMedia M 1297 HiCrome Candida Agar plates for determining the different species of Candida. Chi-square, one-way ANOVA and Tukey HSD Post Hoc test were used as statistical test.

Results: The Gram staining was positive in oral swabs of 14 (28\%) participants in OSMF group and 20 (40\%) participants in OL group. Similarly, the oral swabs in $28 \%$ and $40 \%$ of the participants from OSMF and OL group were positive, when cultured in Sabouraud's Dextrose Agar medium. The positive specimens on culturing in HiMedia M 1297 HiCrome Candida Agar plates detected Candida albicans and non albicans significantly $(\mathrm{p}<0.001) \mathrm{n}$ OSMF and OL group.

Conclusions: The Candida albicans and non albicans are associated with OSMF and OL. The findings of our study may act as diagnostic and prognostic marker, although role of candida in carcinogenesis is still a hypothesis and requires more research.

Keywords: Candida albicans; Candida non albicans; Malignant transformation; Oral leukoplakia; Oral potentially malignant disorders; Oral submucous fibrosis
2 Department of Plastic and

Reconstruction surgery, Harvard Medical

School, Boston, Chinese PLA General

Hospital, Beijing, P.R.China

3 Division of Oral Medicine, Brigham and

Women's Hospital, Harvard Medical

School, Boston, USA

*Corresponding author:

Naman Rao

Đ naman_rao@hms.harvard.edu

Division of Oral Medicine, Brigham and Women's Hospital, Harvard Medical School, Boston, USA.

Tel: 201-913-4129

Citation: More C, Peter R, Nishma G, Chen Y, Rao N (2018) Association of Candida species with Oral Submucous Fibrosis and Oral Leukoplakia: A Case Control study. Ann Clin Lab Res Vol.6 No.3: 248

\section{Key Message}

The study is commenced after a strong clinical experience.

\section{Introduction}

Oral Candidiasis is the most common human fungal infection. It is an important sign of impaired defence mechanism. The candida organism is a part of oral flora and an opportunistic pathogen. The candidal infection arises from an endogenous commensal strain [1,2]. Candida albicans is the most common species of oral cavity, present in both healthy and diseased. The non albicans associated with oral infections are, $C$. kefyr, $C$. krusei, C. dubliniensis, C. guilliermondii, C. glabrata, C. tropicalis and C. Parapsilosis $[1,3,4]$.

The ability of Candida albicans to colonize, penetrate and damage host tissues, depends on an imbalance between the virulence and host defence. The presence of Candida in the mouth along with epithelial changes may predispose individuals to Candidal infection. The oral epithelial changes like atrophy, hyperplasia and dysplasia may compromise the mucosal barrier and facilitate 
candidal invasion. Thus, candida plays an important role in the etio-pathogenesis of oral precancerous lesions [1,3,5]. Candida creates an environment conducive to cell proliferation thereby leading to clonal expansion of genetically altered cells [5-7].

It is hypothesized that Candida species are involved in oral carcinogenesis by elaborating the nitrosamine compounds and by activating specific proto-oncogenes. The oral malignancy is always preceded by Oral Potentially Malignant Disorders (OPMD's) [8-10]. The lesions like Oral Submucous Fibrosis (OSMF) and Oral Leukoplakia (OL) are most commonly seen in South Asia and South East Asian countries [11,12]. The aetiology for these lesions are multifactorial and the Candida organism is thought to be associated with them $[1,2,13]$. The present study was intended to determine the incidence and association of candida species with Oral submucous fibrosis and OL and to correlate the Candida non albicans.

\section{Materials and Methods}

A case control study was undertaken in 150 participants, which were divided into three groups i.e. Group I- OSMF (50), Group IIOL- Homogenous and non-homogenous (50) and Group III- Age and Sex matched Healthy controls (50). The OL was classified as per WHO [14] and the staging of OSMF was as per More et al. classification [5]. The permission to undertake the present study was obtained from Sumandeep Vidyapeeth Institutional Ethics Committee, with vide letter no SVIEC/ON/Dent/RP/16006 dtd. 14/03/16.

Each participant was enrolled after obtaining written consent. The questions were asked to each participant's related to vital statistics, past and present medical and dental history and history of harmful oral habits. The outcome of the same was recorded. Further, a smear or swab was obtained with the help of sterile cotton swab (HIMEDIA PW 0003), either from the site of lesion or from normal mucosa (in healthy controls). Each specimen was subjected for Gram staining to determine the presence of yeast cells. The specimen was inoculated on Sabouraud's dextrose agar for $24-48$ hours at $37^{\circ} \mathrm{C}$ for growth of candida.

The Positive cultured Candida samples were further analyzed by using HIMEDIA M 1297 HiCrome Candida Agar plates for determining the different species of Candida (albicans and non albicans). The Agar plates were incubated for 24-48 hours at $30^{\circ} \mathrm{C}$. The plates were examined for morphology and color of the candida species colony. The appearance of candida species on Crome agar are mentioned in Table 1. The collected data was statistically analyzed by using Statistical Package for the Social Sciences (SPSS) Software (version 17) and the test applied were Chi-square, one-way ANOVA and Tukey HSD Post Hoc test.

\section{Result}

In the present study, 150 participants were equally divided into three groups- OSMF, OL and Healthy controls. The participant's age ranged from 19 to 74 years, with a mean age of $35.47+14.89$ years (Table 2). In OSMF group, 07 (14\%) participants were in Stage I (05 males and 02 females), 06 (12\%) were in stage II (06 males), 27 (54\%) were in Stage III (18 males and 09 females), 10 $(20 \%)$ cases were in stage IVA (07 males and 03 females). In OL group, 35 (70\%) cases had homogenous type (31 males and 04 females), 15 (30\%) had non-homogenous type (12 males and 03 females). The OL were seen, either unilateral (62\%) or bilateral $(38 \%)$ in the oral cavity, involving right buccal mucosa in 13 (26\%) cases, left buccal mucosa in $10(20 \%)$ cases, left and right buccal mucosa in $14(28 \%)$ cases, right commissural area in 04 (8\%) cases, left commissural area in $04(8 \%)$ cases, right and left commissural area in 05 (10\%) cases (Figure 1).

In OSMF group, the minimum and maximum age of participants was 20 and 65 years respectively, with a mean age of $36.04+12.419$. In OL group, the minimum and maximum age of participants was 22 and 74 years respectively, with a mean age of 46.26+14.283. In Healthy controls, the minimum and maximum age of participants was 19 and 65 years respectively, with a mean age of $24.12+8.133$. There was significant $(p<0.001)$ difference between the age groups in all the three groups (One way ANOVA test) (Table 3).

In OSMF group, 32 (64\%) participants had habit of chewing Gutkha, 09 (18\%) participants had habit of chewing plain/flavored arecanut and 09 (18\%) participants had habit of chewing Mawa. In OL group, 29 (58\%) participants had habit of chewing and/or smoking tobacco, 07 participants (14\%) had habit of chewing Gutkha, 14 (28\%) had habit of chewing plain/flavored arecanut. The probability ' $p$ ' value was obtained $(0.476)$ using Pearson's chi square test, which was not significant $(p>0.001)$.

The oral smear/swab of each participant when subjected to Gram staining, 14 (28\%) participants in OSMF group were positive, 20 (40\%) participants were positive in OL group and all the healthy controls were negative (Figure 2 ). The ' $p$ ' value was significant $(p<0.001)$ on using Pearson's chi square test. The oral swab when cultured in Sabouraud's Dextrose Agar medium, it was observed that, in OSMF and OL group, 14 (28\%) participants and $20(40 \%)$ participants were positive respectively. The probability ' $p$ ' value was obtained using Pearson's chi square test, which was significant $(p<0.001)$ (Table 4).

Table 1 Showing color coding for candida species.

\begin{tabular}{|c|c|}
\hline Color & Species \\
\hline Green & C. albicans \\
\hline Blue & C. tropicalis \\
\hline Purple & C. krusei \\
\hline Whitish pink & C. glabrata \\
\hline Cream & C. parapsilosis \\
\hline
\end{tabular}

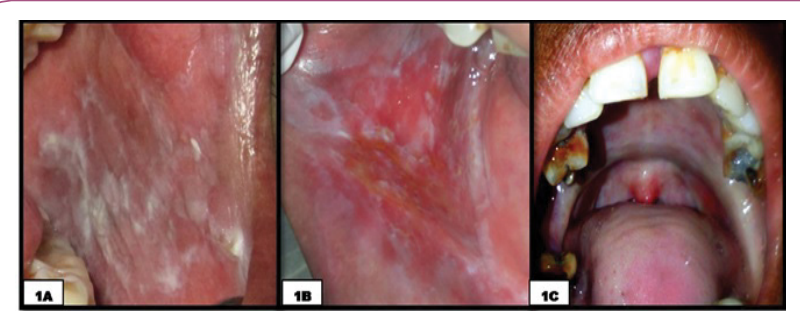

Figure 1 Shows- (A) Diffused type of Oral leukoplakia on left buccal mucosa. (B) Non homogenous type (Erythroleukoplakia) on right buccal mucosa. (C) Oral submucous fibrosis involving posterior part of the oral. 
The positive specimens were cultured in HIMEDIA M 1297 HiCrome Candida Agar plates for detecting Candida species through colored colonies. In OSMF group, out of 14 positive Candida samples, 10 (71.43\%) had green color colonies ( $C$. albicans), 03 (21.43\%) had blue color colonies (C. tropicalis), 01 (7.14\%) had purple colour colonies (C. krusei). In OL group, out of 20 positive Candida samples, 11 (55\%) had green colour colonies (C. albicans), 8 (40\%) had blue color colonies (C. tropicalis) and $01(5 \%)$ had purple colour colonies (C. krusei). The probability ' $\mathrm{p}$ ' value was significant $(p<0.001)$ on using Pearson's chi square test (Figure 3 and Table 5).

It is significant to note here that, out of 14 positive Candida specimens in OSMF group, 10 had habit of chewing Gutkha, 03 had Mawa chewing habit and 01 had Arecanut chewing habit. Similarly, in OL group out of 20 positive candida specimens, 11 had Tobacco chewing habit, 06 had Bidi smoking habit and 03 had habit of Mawa chewing.

\section{Discussion}

OPMD's are indicators of risk for likely future malignancies. It is now clear that even the clinically 'normal' appearing mucosa in a patient harbouring a precancerous lesion may have dysplasia on the contralateral anatomic site or molecular aberrations in other oral mucosal sites suggestive of a pathway to malignant transformation, and that cancer could subsequently arise in apparently normal tissue. The pathogenesis for these lesions is multifactorial. Amongst the OPMD's, the most common are $\mathrm{OL}$ and OSMF.

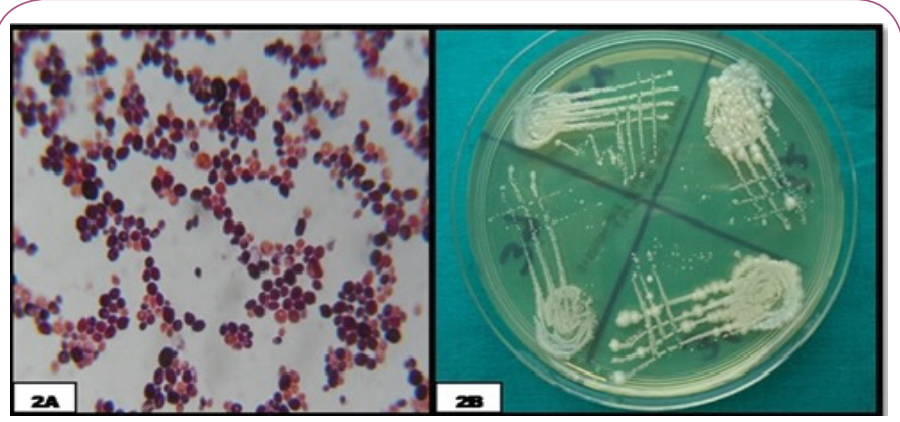

Figure 2 Shows- (A) Photomicrograph showing yeast cells after gram staining. (B) Sabouraud's dextrose agar culture showing positive candida.

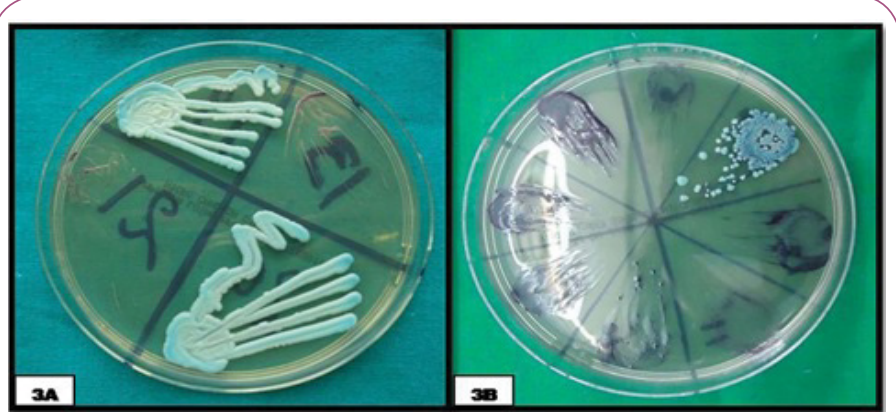

Figure 3 Shows HiCrome agar plate (A) Candida albicans and (B) Candida non albicans.
Various factors trigger the disease process by causing mucosal changes at cellular levels and at different sites of oral cavity. The use of tobacco, arecanut and alcohol are considered as the most important high-risk determinants for OPMD's $[5,14]$. The synergistic effects of these factors have high carcinogenic potency. The other low risk factors like chronic irritation, galvanism, nutritional deficiency, syphilis, infections (bacterial, fungal, viral), genetic susceptibility, hormonal disturbances, ultraviolet light radiation, autoimmunity, etc. are also associated with these lesions and have synergistic role with high risk factors in carcinogenesis $[15,16]$.

Candida is a commensal inhabitant of oral mucosa in healthy individuals. It plays an important role in oral carcinogenesis through production of components like endogenous nitrosamine, higher concentrations of acetaldehyde, oligosaccharide and lectin. Candida albicans is the most common organism found in the oral cavity whereas Candida non albicans are rarely seen $[3,16]$. Candida albicans is highly infectious amongst the candida species. The Candida non albicans lack totally or partially the virulence factor. In recent times, the non-candida albicans species are isolated from oral mucosa in varying colonies in various oral lesions specially OPMD's. These non-candida albicans are resistant to the commonly used antifungal agents. The probable role of non-candida albicans in the neoplastic transformation of OSMF and OL, is still obscure $[4,6]$.

The present study was conducted with a larger sample size, on three groups- OSMF, OL and Healthy controls. The study had $65.3 \%$ males and the age of participants ranged from 19 to 74 years, with a mean age of $35.47+14.89$. The minimum age of participants in OSMF and OL was 20 years and 22 years respectively, which was statistically significant $(p<0.001)$. Ourthese findings matched with the findings of Goel et al. [17], Ali et al. [18], Kalbande et al. [19] and did not match with the findings of Reddy et al. [20], Kumar et al. [21]. It is pertinent to note here that the age of having lesions like OL and OSMF have dropped down from forties to twenties. The reasons for this may be easy availability and upsurge in the intake of tobacco, arecanut and alcohol related products at early age.

In our study, the maximum participants had stage III OSMF (54\%) and homogenous leukoplakia (70\%); and the common site of occurrence was buccal mucosa. This finding was similar to the studies of Sahay et al. [16], Hebbar et al. [22], Dany et al. [23], Singh et al. [24]. We distinctly observed that $64 \%$ of OSMF participants had habit of chewing Gutkha other than chewing habits like plain/flavored arecanut and mawa. Also, in $58 \%$ of OL participants, the use of tobacco in chewing and/or smoking form was observed ( $p>0.001)$. Our, this finding was similar to the findings of Sahay et al. [16], Saigal et al. [25], More et al. [26]. Our study result confirms the strong association of chewing gutkha with OSMF and tobacco with $\mathrm{OL}$, in the initiation and progression of the lesion, although the type, brand, duration and frequency of these habits will also have a major role to play.

The presence and association of candida in our study was ruled out by various investigations using Gram stain, Sabouraud's dextrose Agar medium and HiCrome agar medium. In our study, $28 \%$ and $40 \%$ of the oral swabs/smear were positive in OSMF and 
Table 2 Distribution of participants based on gender.

\begin{tabular}{|c|c|c|c|c|c|c|}
\hline \multicolumn{7}{|c|}{ Gender } \\
\hline \multirow[t]{2}{*}{ Group } & \multicolumn{2}{|c|}{ Male } & \multicolumn{2}{|c|}{ Female } & \multirow[t]{2}{*}{$\mathbf{n}$} & \multirow[t]{2}{*}{$\%$} \\
\hline & $\mathbf{n}$ & $\%$ & $\mathbf{n}$ & $\%$ & & \\
\hline OSMF(I) & 36 & 72.0 & 14 & 28.0 & 50 & 100 \\
\hline $\mathrm{OL}(\mathrm{II})$ & 44 & 88.0 & 6 & 12.0 & 50 & 100 \\
\hline Healthy controls (III) & 18 & 36.0 & 32 & 64.0 & 50 & 100 \\
\hline Total & 98 & 65.3 & 52 & 34.7 & 150 & 100 \\
\hline \multicolumn{7}{|c|}{ Note: $\mathrm{n}$ - frequency, \%- percentage } \\
\hline
\end{tabular}

Table 3 Age wise distribution of participants (One-way ANOVA test).

\begin{tabular}{|c|c|c|c|c|}
\hline Group & $\mathbf{n}$ & Minimum Age (in yrs) & Maximum Age (in yrs) & Mean \pm SD \\
\hline OSMF (I) & 50 & 20 & 65 & $36.04 \pm 12.41$ \\
\hline OL (II) & 50 & 22 & 74 & $46.26 \pm 14.28$ \\
\hline HC (III) & 50 & 19 & 65 & $24.12 \pm 8.13$ \\
\hline Total & 150 & 19 & 74 & $35.47 \pm 14.89$ \\
\hline
\end{tabular}

Note: OSMF- Oral Submucous Fibrosis, OL- Oral Leukoplakia, HC- Healthy Controls, HS- Highly Significant, n- Frequency

Table 4 Distribution of Participants based on Gram staining and Sabouraud's medium culture for Candida (Pearson's chi square test).

\begin{tabular}{|c|c|c|c|c|c|}
\hline \multirow[t]{2}{*}{ Particulars } & \multicolumn{3}{|c|}{ Group } & \multirow{2}{*}{$\begin{array}{c}\text { Total } \\
(n=150)\end{array}$} & \multirow[t]{2}{*}{ p-value } \\
\hline & $\operatorname{OSMF}(I)(n=50)$ & OL (II) $(n=50)$ & $H C(I I I)(n=50)$ & & \\
\hline \multicolumn{5}{|c|}{ Gram staining } & \multirow{3}{*}{$\begin{array}{c}<0.001 \\
\text { HS }\end{array}$} \\
\hline $\begin{array}{c}\text { Positive } \\
(\%)\end{array}$ & $\begin{array}{c}14 \\
(36)\end{array}$ & $\begin{array}{c}20 \\
(40) \\
\end{array}$ & $\begin{array}{c}0 \\
(00)\end{array}$ & $\begin{array}{c}34 \\
(22.7) \\
\end{array}$ & \\
\hline $\begin{array}{c}\text { Negative } \\
(\%)\end{array}$ & $\begin{array}{c}36 \\
(72) \\
\end{array}$ & $\begin{array}{c}30 \\
(60 \%) \\
\end{array}$ & $\begin{array}{c}50 \\
(100) \\
\end{array}$ & $\begin{array}{c}116 \\
(77.3) \\
\end{array}$ & \\
\hline \multicolumn{5}{|c|}{ Sabouraud's medium } & \multirow{3}{*}{$\begin{array}{c}<0.001 \\
\text { HS }\end{array}$} \\
\hline $\begin{array}{c}\text { Positive } \\
(\%)\end{array}$ & $\begin{array}{c}14 \\
(36)\end{array}$ & $\begin{array}{c}20 \\
(40) \\
\end{array}$ & $\begin{array}{c}0 \\
(00)\end{array}$ & $\begin{array}{c}34 \\
(22.7) \\
\end{array}$ & \\
\hline $\begin{array}{c}\text { Negative } \\
(\%)\end{array}$ & $\begin{array}{c}36 \\
(72)\end{array}$ & $\begin{array}{c}30 \\
(60 \%)\end{array}$ & $\begin{array}{c}50 \\
(100)\end{array}$ & $\begin{array}{c}116 \\
(77.3)\end{array}$ & \\
\hline
\end{tabular}

Table 5 Distribution of participants according to candida species on HiCrome candida agar medium (Pearson's chi square test).

\begin{tabular}{|c|c|c|c|c|c|}
\hline \multirow{2}{*}{ Candida Species } & \multicolumn{3}{|c|}{ Group } & \multirow{2}{*}{ Total $(n=150)$} & \multirow{2}{*}{ p-value } \\
\hline & OSMF (I) $(n=50)$ & OL (II) $(n=50)$ & $H C$ (III) $(n=50)$ & & \\
\hline C. albicans (\%) & $10(20)$ & $11(22)$ & $0(0)$ & $21(14)$ & \multirow{4}{*}{$<0.001 \mathrm{HS}$} \\
\hline C. tropicalis (\%) & $03(6)$ & $8(16)$ & $0(0)$ & $11(7.3)$ & \\
\hline C. krusei (\%) & $01(2)$ & $1(2)$ & $0(0)$ & $2(1.3)$ & \\
\hline No species isolated (\%) & $36(72)$ & $30(60)$ & $50(100)$ & 116 (77.3) & \\
\hline
\end{tabular}

OL respectively. All the samples of healthy controls were negative for Gram stain. Our, this finding was similar to the findings of Sahay et al. [16], Dixit et al. [27], Nayak et al. [28]. Similarly, the Sabouraud's dextrose agar, which is widely used as a selective medium for the isolation of Candida and other yeast species from clinical specimens; was used to culture the oral swabs/smear of all the participants. The findings on the culture were similar to the findings of gram staining [28] $(p<0.001)$.

The chromogenic culture medium HIMEDIA M 1297 HiCrome Candida Agar was used for the isolation and direct identification of some of the most clinically important yeast pathogens on the basis of colony color [20]. When the positive specimens were cultured in this medium, In OSMF group, out of 14 positive Candida samples, 10 (71.43\%) had green color colonies ( $C$. albicans), 03 (21.43\%) had blue color colonies (C. tropicalis), 01 (7.14\%) had purple color colonies (C. krusei). In OL group, out of 20 positive Candida samples, 11 (55\%) had green color colonies (C. albicans), 8 (40\%) had blue color colonies (C. tropicalis) and 01 (5\%) had purple color colonies (C. krusei). The probability ' $p$ ' value was significant $(p<0.001)$ on using Pearson's chi square test. Our, this finding matched with the findings of Sahay et al. [16], Dixit et al. [27], Nayak et al. [28].

It is also important to note that the present study encountered candida non albicans quantitatively and qualitatively, which is 
unexpected because it is proved that Candida albicans is the sole organism which is present in the oral cavity. The presence of noncandida albicans in such a significant manner in our study raises suspicion about the change in the oral flora. The reasons for the same may be multifactorial.

The present study has significant observation when the positive candida specimens were correlated with type of habit. It suggested that chewing of Gutkha and tobacco has some relation with the pathogenic growth of candida in the oral cavity. One or more contents of tobacco and arecanut/gutkha may have some role in creating favourable conditions for the candida to adhere to

\section{References}

1 Bakri M, Hussaini H, Holmes A, Cannon R, Rich A (2010) Revisiting the association between candidal infection and carcinoma, particularly oral squamous cell carcinoma. J of Oral Microbio 2(1): 57-80.

2 Anila K, Hallikeri K, Shubhada C, Naikmasur V, Kulkarni R (2011) Comparative study of candida in oral submucous fibrosis and healthy individuals. The Revista Odonto 26(1): 71-76.

3 Nada V, Marija B, Dejan V, Ivana P (2004) Presence of Candida albicans in potentially malignant oral mucosal lesions. Arch Oncol 12(1): 51-54.

4 Sun J, Qi C, Lafleur M, Qi Q (2009) Fluconazole susceptibility and genotypic heterogeneity of oral Candida albicans colonies from the patients with cancer receiving chemotherapy in China. Int J of Oral Sci 1(3): 156-162.

5 More CB, Das S, Patel H, Adalja C, Kamatchi V (2012) Proposed clinical classification for oral submucous fibrosis. Oral Oncol 48(3): 200-202.

6 Birman E, Kignel S, Silveira F, Paula C (1997) Candida albicans: Frequency and characterization in oral cancer (Stage I) from smokers and drinkers. Rev Iberoam Micol 14: 101-103.

7 Scully C (2011) Oral cancer aetiopathogenesis: Past, present and future aspects. Med Oral Patol Oral Cir Bucal 16(3): 306-311.

8 More CB, Pawar R, Rao NR, Shah PH, Gavli NJ (2015) Oral ulcer: An overview with an emphasis on differential diagnosis. Int J Oral Health Sci (4): 1-13.

9 Rao N, Parikh A, Patel A, Parsana H, Dave M (2017) Oral cancer: A review of recent non-invasive diagnositic methods. Int J Curr Res 9(10): 59973-59976.

10 Adalja C, Adalja C, More C, Rao N (2016) Role of stem cell therapy in oral premalignant and malignant lesions. Int J Curr Res 8(12): 4388043883.

11 More C, Shilu K, Gavli N, Rao N (2018) Etiopathogenesis and clinical manifestations of oral submucous fibrosis, a potentially malignant disorder: An update. Int J Curr Res 10(7): 71816-71820.

12 More C, Shah P, Rao N, Pawar R (2015) Oral submucous fibrosis: An overview with evidence-based management. Int J Oral Health Sc 3(3): 40-49.

13 Mehrotra R, Yadav S (2006) Oral squamous cell carcinoma: Etiology, pathogenesis and prognostic value of genomic alterations. Ind Jou of Can 43(2): 60-66.

14 Greenberg M, Glick M (2008) Burket's oral medicine diagnosis and treatment. 11th Edition, Elsevier, Red \& White lesion of the oral mucosa 4: 77-106. the oral mucosa especially on the diseased area like OSMF, OL etc. The studies shall be undertaken in near future on the correlation between the type of habit and candida species.

\section{Conclusion}

The present study has confirmed the significant association between the stage/type of lesion and candidal growth; and type of habit with colonization of the Candida species. The significant presence of rare non-candida albicans species in OSMF and OL, raises suspicion about its role in the progression and malignant transformation.

15 More C, Gupta S, Joshi J, Varma S (2012) Classification system for oral submucous fibrosis. Jou of Indian Acad of Oral Med and Radio 24(1): 24-29.

16 Sahay A (2012) Occurrence of Candida albicans in oral leukoplakia: A clinical and histological evaluation. Ind J of Dent Edu 5(3): 137-145.

17 Goel S, Ahmed J, Singh MP, Nahar P (2010) Oral submucous fibrosis: A clinico-histopathological comparative study in population of Southern Rajasthan. J Carcinogene Mutagene 1(2): 108-111.

18 Ali FM1, Aher V, Prasant MC, Bhushan P, Mudhol A, et al. (2013) Oral submucous fibrosis: Comparing clinical grading with duration and frequency of habit among areca nut and its products chewers. J Cancer Res Ther 9(3): 471-476.

19 Kalbande AB, Khakse GM, Priya D, Tamgadge PB (2013) Epidemiological study of oral submucous fibrosis in Yavatmal district. IJRTSAT 6(1): 38-40.

20 Reddy V, Wanjari PV, Reddy NB, Reddy P (2011) Oral submucous fibrosis: Correlation of clinical grading to various habit factors. International Journal of Dental Clinics 3(1): 21-24.

21 Kumar Kiran, Saraswathi TR, Rangnathan K, Devi Uma M, Elizabeth Joshua (2007) Oral submucous fibrosis: A clinic histopathological study in Chennai. IJDR 18(3): 106-111.

22 Hebbar P, Pai A, Sujatha D (2013) Mycological and histological associations of Candida in oral mucosal lesions. J of Oral Sci 55(2): 157-160.

23 Dany A, Kurian, Shanmugam (2011) Association of candida in different stages of oral leukoplakia. J of Ind Acad of Oral Med and Radio 23(1): 14-16.

24 Singh K, Gupta A, Rajan S, Padmavathi B, Mamatha G, et al. (2014) Correlation of presence of candida and epithelial dysplasia in oral mucosal lesions. J of Clin and Diag Res 8(10): 31-35.

25 Saigal S, Bhargava A, Mehra S, Dakwala F (2011) Identification of Candida albicans using different culture medias and its association in potentially malignant and malignant lesions. Contemp Clin Dent 2(3): 188-193

26 More C, Asrani M, Patel H, Adalja C (2010) Oral submucous fibrosis: A hospital based retrospective study. Pearldent 1(4): 25-31.

27 Dixit S, Goswami S, Dive A (2012) Determination, distribution and phenotypic differentiation of Candida: Study in oral precancer and oral cancer. Ind J of Dent Re and Rev pp: 1-4.

28 Nayak S, Nirmal M, Gokul S, Adarsh H, Manjunath N (2012) Analysis of Candidal carriage in oral submucous fibrosis: A case control study. Int J of Dent Upd 2(1): 10-15. 